\title{
Sweating the Small Stuff: Pitfalls in the Use of Radiation Detection Instruments*
}

\author{
Jennifer Lynne Prekeges, CNMT \\ Bellevue College, Bellevue, Washington
}

\begin{abstract}
Nuclear medicine technologists use nonimaging radiation detection instruments on a daily basis and routinely assess their performance in several ways. These instruments are simple to operate compared with imaging instruments but are also susceptible to misuse. After reviewing the theoretic basis for common mistakes and the importance of routine quality control, this continuing education article discusses pitfalls in the operation of dose calibrators, survey meters, scintillation detectors, and semiconductors. The article also reviews quality control procedures and ways in which these can be performed incorrectly. The goal of the article is to help technologists to spot and correct problems before they lead to errant results.
\end{abstract}

Key Words: radiation detectors; radiation measurement; quality control

J Nucl Med Technol 2014; 42:81-91

DOI: 10.2967/jnmt.113.133173

\section{$\mathbf{T}$} he practice of nuclear medicine relies not only on $\gamma$ cameras and PET tomographs but also on a variety of radiation detection instruments. Dose calibrators are used to measure dosage in activity units, and survey meters such as Geiger counters indicate radiation field strengths. Radioactive samples can be measured with scintillation detectors, and radioactivity in body organs can be assessed using organ probes made with either a scintillation crystal or semiconductor material. None of these small instruments provides an image; they only generate numbers. One of the major responsibilities of a nuclear medicine technologist is to ensure that these numbers correctly represent the amount of radioactivity present in the source and the geometry of the measurement.

Quality control (QC) procedures are intended to verify instrument function and ensure accuracy and precision. But even beyond actual instrument problems that should be

\footnotetext{
Received Sep. 25, 2013; revision accepted Jan. 30, 2014.

For correspondence or reprints contact: Jennifer Lynne Prekeges, Bellevue

College, 3000 Landerholm Circle SE, Bellevue, WA 98007-6484.

E-mail: jennifer.prekeges@bellevuecollege.edu

*NOTE: FOR CE CREDIT, YOU CAN ACCESS THIS ACTIVITY THROUGH THE SNMMI WEB SITE (http://www.snmmi.org/ce_online) THROUGH JUNE 2016.

Published online Mar. 13, 2014.

COPYRIGHT (C 2014 by the Society of Nuclear Medicine and Molecular Imaging, Inc.
}

identified in QC, there are many ways to obtain incorrect results when using these instruments. The goal of this article is to call these potential pitfalls to our attention. We will first review the general categories of pitfalls, which include distance and geometry, extraneous activity, and incorrect energy window settings. Each instrument will then be addressed individually, including a review of the applicable QC procedures and discussion of how errors in procedure performance can result in incorrect results.

Several references provide broader explanations of the components and operating principles of small radiation instruments (1-4). The reader is referred to these for additional background information on each instrument or QC procedure. Licensing and accrediting bodies, such as the Nuclear Regulatory Commission, the American College of Radiology, the Society of Nuclear Medicine and Molecular Imaging, and the Intersocietal Commission on the Accreditation of Nuclear Laboratories, publish standard-of-practice QC guidelines (5-7). Protocols and mathematic formulas for QC tests are described in various references $(8,9)$.

\section{FACTORS AFFECTING MEASUREMENT OF RADIOACTIVITY}

The factors that determine the value obtained when measuring a radiation source include the calibration factors that adjust the instrument's readout, the amount of the radioactive substance to be measured or the strength of the radiation field, the types and energies of the radiations contributing to the measurement, the distance between the detector and the radiation source, and the material containing or shielding the radiation source. Most mistakes in radioactivity measurements are related to one of these factors. Before getting into the potential for errors associated with individual radiation detectors, we will consider these factors from a more general perspective.

\section{Distance and Geometry}

The inverse-square law is a basic physical principle of radiation detection. According to this mathematic relationship, the intensity of a radioactive source varies inversely with the square of the distance to the detector. The inversesquare law is quantitatively correct only to the extent that the radioactive source looks like a point source. The distance must be considerably larger than the source dimension in order for this to be true. For example, measurement of 
a spheric source that is $2 \mathrm{~cm}$ in diameter will not exactly follow the prediction of the inverse-square law until it is 200 $\mathrm{cm}(2 \mathrm{~m})$ or more from the radiation detector. At closer distances, the size of the source causes the detector to measure higher radiation levels than predicted, because the source irradiates the detector from a wide angle. So it is important to be aware that while the principle of decreasing readings with increasing distance always applies, the exact measurements may not track with the inverse-square law at close range.

Even small changes in geometry or source orientation may cause a radiation reading to vary. A key tenet of small instrument QC is to maintain the same geometry for each repetition of a given test. Training and the use of diagrams are two ways to accomplish this. As one example of the importance of geometry, consider that a dose calibrator constancy measurement may be affected by placing the reference source into the dipper upside down rather than right-side up. Geometry considerations become crucial when working with an instrument that has directionality, such as an organ probe. This is especially true when the radioactivity in the reference source is not uniformly distributed.

The inverse-square law assumes that there is nothing except air between the source and the detector. The presence of shielding material, even the source container, may change the measured value. In some situations, such as the dose calibrator linearity test, we use shielding material to simulate changes in the quantity of radioactivity, to verify proper function. But most measurements require that the space between the source and the detector be free of any material that might absorb radiation.

\section{Extraneous Sources of Radiation}

Another basic assumption in radiation measurement is that only the specific radiation source in question is being measured. Radiation is found throughout the environment, and we must often account for its presence by measuring and subtracting background radiation levels. Failure to subtract background is a common source of error in small instrument situations.

A nuclear medicine department contains multiple radiation sources at any given time, including radiopharmaceutical syringes, radioactive patients, QC sources, radioactive waste, and (these days) x-ray tubes for CT studies. One must ensure that none of these sources are accidentally included in a radiation measurement, because any extraneous radiation will produce a falsely high reading. Considerations should also include big-picture questions such as department layout.

A particularly difficult problem in the category of extraneous sources is that of radioactive contamination. A contaminated instrument will not give a correct reading of the intended radioactive source until the contamination is gone. Although contamination can sometimes be removed by cleaning, in many situations the only solution is to wait for decay.

\section{Energy Window}

Scintillation and semiconductor radiation detectors are sophisticated enough to be able to identify the energy of photon radiations. This is beneficial, in that it allows discrimination of photons of different energies, but it also necessitates the proper choice of energy window to detect the desired photons. Use of an incorrect energy window will obviously produce a reading that is incorrect-in almost all cases lower than it should be. With a $\gamma$ camera, an image taken with the wrong energy window clearly looks different from the same image using the correct energy window (2). With a small instrument such as a scintillation detector, however, there is no image to go by, only a number. This makes it even more important that the operator verify the energy window before making the measurement.

\section{ROUTINE QC}

QC has always been a major emphasis in the practice of nuclear medicine but has taken on greater importance in these days of laboratory accreditation. It is only when we know our instruments are working properly that we can attach real meaning to clinical or radiation safety measurements. QC is important for the welfare of both patients (e.g., verification that a radiopharmaceutical dosage is correct) and technologists (e.g., measurement of the scattered radiation from operation of a SPECT/CT or PET/CT camera). Hence the discussion of pitfalls in radiation measurement will include a focus on the QC procedures required for each instrument.

Small instruments are generally tested with a long-lived reference source. Several aspects of the choice of reference sources deserve careful attention. First, if a laboratory desires to measure the accuracy of its dose calibrator or calibrate its survey meters, the source or sources used must be traceable to the National Institute of Standards and Technology. This ensures that the quantity of the source activity is known to the degree required for these tests. Second, reference sources have different photon energies, and one should choose surrogate radionuclides with energies that simulate the photons encountered in the clinical setting (Table 1). Third, whereas they are all long-lived compared with radionuclides administered to patients, these sources do have finite lifetimes that make regular replacement necessary. Finally, reference sources require their own QC. They must be inventoried and leak-tested semiannually, and they need to be disposed of properly.

\section{DOSE CALIBRATOR}

A dose calibrator (Fig. 1) is a gas-filled detector, in which the cylindrically shaped, pressurized gas chamber surrounds the measuring space. Interactions of photons in the gas cause ionizations, generating free electrons that are detected as electric current. A conversion factor specific to the isotope being measured converts the current reading to a value in units of radioactivity. Radioactive sources are 
TABLE 1

Reference Sources for Small Instruments

\begin{tabular}{|c|c|c|c|c|}
\hline Radionuclide & Half-life & $\begin{array}{l}\text { Principal } x \text { - or } \\
y \text {-ray energy }\end{array}$ & Surrogate for... & Common uses \\
\hline${ }^{137} \mathrm{Cs}$ & $30 y$ & $662 \mathrm{keV}$ & & $\begin{array}{l}\text { Dose calibrator constancy, accuracy } \\
\text { Scintillation detector calibration, } \\
\text { energy resolution }\end{array}$ \\
\hline${ }^{68} \mathrm{Ge}$ & $287 d$ & $\begin{array}{l}511 \mathrm{keV} \text { (from }{ }^{68} \mathrm{Ga} \\
\text { daughter) }\end{array}$ & $\begin{array}{l}\text { Positron-emitting } \\
\text { radionuclides }\end{array}$ & $\begin{array}{l}\text { Well counter constancy, accuracy } \\
\text { Dose calibrator constancy }\end{array}$ \\
\hline${ }^{133} \mathrm{Ba}$ & $10.7 y$ & $356 \mathrm{keV}$ & 131| & $\begin{array}{l}\text { Dose calibrator constancy } \\
\text { Scintillation detector efficiency }\end{array}$ \\
\hline${ }^{57} \mathrm{Co}$ & $272 d$ & $122 \mathrm{keV}$ & 99mTc & $\begin{array}{l}\text { Dose calibrator constancy } \\
\text { Scintillation detector energy } \\
\text { resolution } \\
\text { Semiconductor probe constancy }\end{array}$ \\
\hline
\end{tabular}

Adapted from Zanzonico (8).

placed into the measuring space via a dipper, which is designed to ensure consistent positioning of the source for measurement. This brief description of its use immediately suggests several ways in which an incorrect value can be produced. For example, the wrong isotope button can be chosen, causing an incorrect conversion factor to be applied; the source may be incorrectly placed in the dipper; or the dipper can become contaminated, at which point it acts as an extraneous source that is included in the measurement of the desired source.

Dose calibrators measure the average current produced in the gas chamber, which takes a finite amount of time to achieve a stable measurement. This in turn results in a lower limit for activity measurement in most dose calibrators of about $3,700 \mathrm{~Bq}(0.1 \mu \mathrm{Ci})$. At very low activities, as much as $20 \mathrm{~s}$ may be required for the readout to stop fluctuating. Consequently, if a high level of accuracy is required for activities of less than about $750 \mathrm{kBq}$ $(\sim 20 \mu \mathrm{Ci})$, a well counter measurement (after dilution) is preferred (10). Even for doses in the $\mathrm{MBq}(\mathrm{mCi})$ range, several seconds may still be needed for the readout to settle on a single value.

Dose calibrators are most commonly found in a nuclear medicine hot lab, which has any number of radiation sources. The dose calibrator is therefore surrounded by a lead shield, so that only photons coming from within the measuring space contribute to the reading. The shield also causes photons from the source in the measuring space to backscatter into the gas chamber. Isotope conversion factors are determined with the shield in place $(10,11)$. If the shield is not in place around the gas chamber, readings will be affected by other radiation sources and by the lack of backscatter and will be incorrect. The manufacturer's dipper and liner also must be used; these may not be interchangeable from one dose calibrator to the next.

Given its use in measuring patient dosages, the dose calibrator is a vital piece of equipment in the nuclear medicine department, and ensuring its proper function is essential. Daily, quarterly, and annual QC testing is required and must be properly performed and documented. We will consider each test in light of the requirements of the test and errors that affect the results.

\section{Constancy}

Constancy testing is performed daily, before the measurement of patient doses, to ensure that the dose calibrator

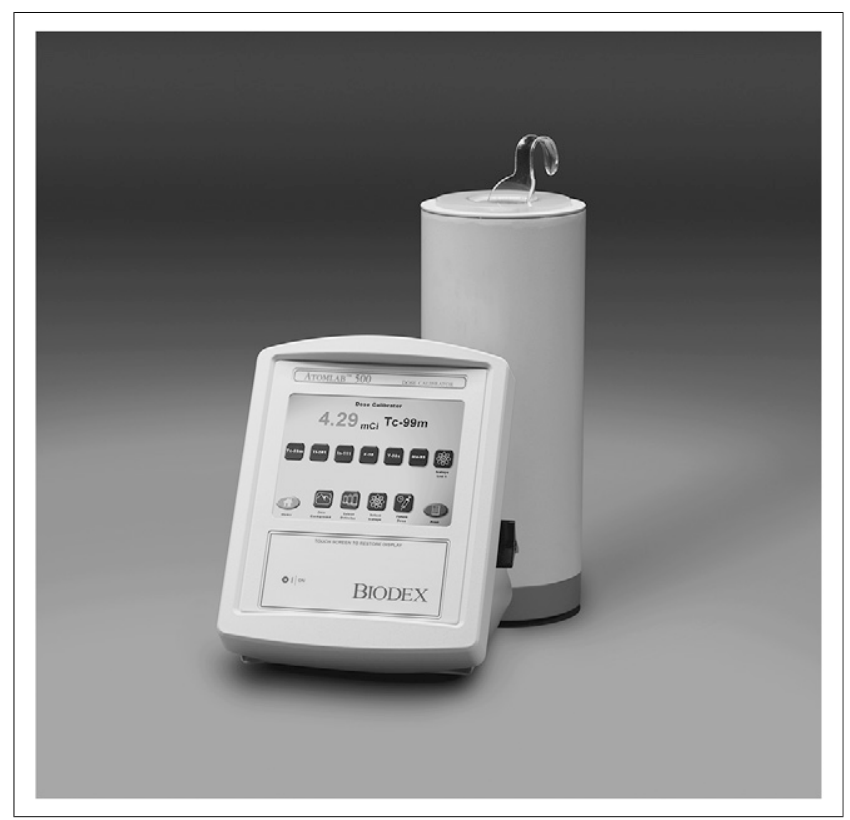

FIGURE 1. Dose calibrator. Electronics module almost always sits separately from cylindric ionization chamber, as shown in this figure. lonization chamber is surrounded by lead shield, which blocks external radiation from entering and also causes photons from source being measured to be backscattered into gas space. Dipper handle can be seen coming out of measurement space. (Photo courtesy of Biodex Medical Systems, Inc.) 
is responding correctly to a long-lived source in a consistent manner. The constancy test in a modern, electronic dose calibrator includes a check of the electrometer sensitivity (auto-zero), measurement and automatic subtraction of background, and a comparison of chamber voltage with the factory-established value. The long-lived source, commonly ${ }^{137} \mathrm{Cs}$ or ${ }^{57} \mathrm{Co}$, is then placed in the dipper and a reading obtained. If this source is National Institute of Standards and Technology-traceable, the test can be considered an accuracy test (8); if not, the goal is simply to determine whether the day's reading is consistent with prior readings of this source. As a general rule, the measured value should be within $\pm 5 \%-10 \%$ of the expected value for the source (manufacturer's recommendations and state regulations vary in their requirements). Many departments check readings of the reference source with other commonly used isotope buttons, and these also should produce consistent values from one day to the next. Day-to-day constancy measurements should vary only within a narrow range, with the value slowly decreasing over time because of decay of the source. The geometry of the source placement is important-it should be centered and upright in the dipper. If the source is upside down or tipped on its side, both the activity measurement and the day-to-day variation can change.

\section{Accuracy}

Accuracy testing of dose calibrators generally requires 2 different National Institute of Standards and Technologytraceable radionuclide sources, one of which should have photon energy of 100-500 keV. As with the constancy measurement, the source geometry must be consistent and the correct isotope button must be used. Most dose calibrators have a system test (an internal test of electronic components) that should be run in conjunction with accuracy testing. Documentation of accuracy testing is often requested by regulatory and accrediting agencies. A common problem with this test is not performing it within the required (usually annual) interval.

\section{Linearity}

Constancy and accuracy testing involve only 1 or 2 activity levels. The linearity test evaluates the response of the dose calibrator at a variety of activity levels, ranging from the largest values measured with the dose calibrator down to $\mathrm{kBq}(\mu \mathrm{Ci})$ levels. Although linearity can be performed by measuring the decay of a short-lived source such as ${ }^{99 \mathrm{~m}} \mathrm{Tc}(2,9)$, it is more quickly accomplished using a series of lead tubes that are placed around a highactivity source to simulate lower and lower activity levels. These have trade names such as Lineator (Biodex Medical Systems) and Calicheck (Fluke Biomedical) (Fig. 2). Initially, the operator determines the calibration factor for each combination of tubes. For subsequent linearity testing, the readings produced are multiplied by the corresponding calibration factors and the resulting values compared with

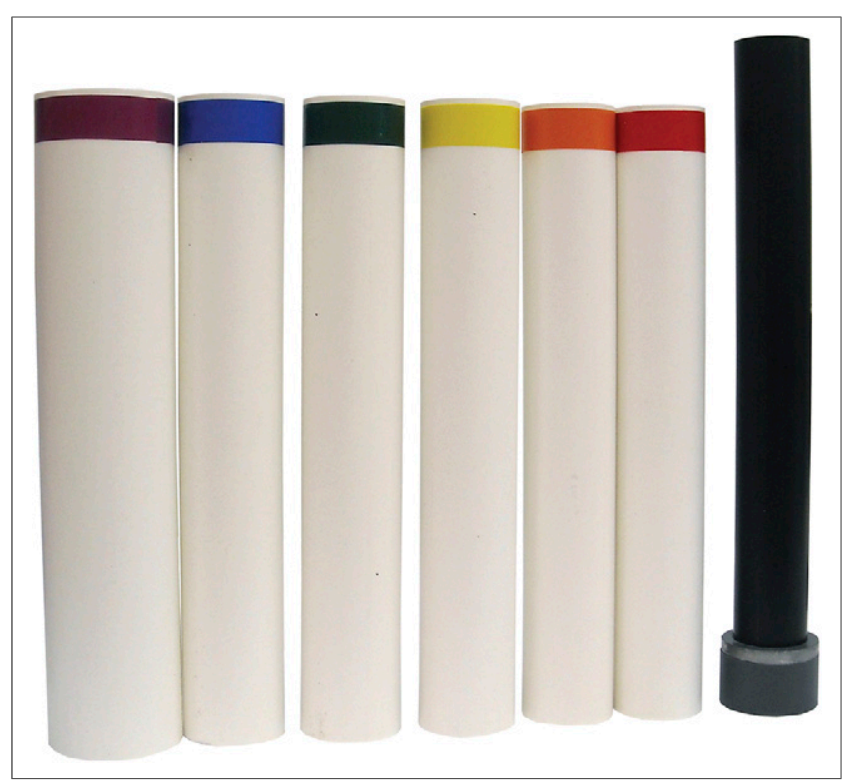

FIGURE 2. Linearity attenuation shields. Source is placed in black tube, which is in turn placed within measuring space of dose calibrator. Other tubes, which have colored bands on upper edge, are then placed into dose calibrator around black tube. Known attenuation factors of each combination of tubes allow operator to simulate activity levels over wide range, thus assessing dose calibrator's ability to measure accurately at different activity levels. (Photo courtesy of Capintec, Inc.)

the expected reading (Table 2). Consistency in geometric placement of the source is crucial, as is the application of the correct calibration factor for each tube or combination of tubes.

\section{Geometry}

For a dose calibrator, geometry testing encompasses volume, depth of placement in the detector chamber, and source container considerations. The effect of volume is tested by recording the value of $1 \mathrm{~mL}$ of a liquid radioactive source such as ${ }^{99 \mathrm{~m}} \mathrm{Tc}$ and then adding liquid to the source in $1-\mathrm{mL}$ increments up to the maximum volume of the container. This test must be done carefully so as not to lose any of the initial activity. The effect of depth is assessed by measuring radioactivity in a vial at different positions within the measuring space, from bottom (resting on the dipper) to top. As more and more photons travel at angles that allow them to escape the measuring space, there will come a point at which the reading decreases below the allowed $\pm 10 \%$ value. This point should be noted, and measurements should not be made when sources rise above it.

The source container issue relates to $\beta$-emitting and low-energy $\gamma$-ray sources. Low-energy photons are easily absorbed by low-atomic-number materials such as glass. A plastic syringe will attenuate some of these photons, but not as many as a glass vial. $\beta$ particles emitted by a radionuclide are not measured directly in a dose calibrator, because they 
TABLE 2

Example of Linearity Determination Using Attenuation Tubes

\begin{tabular}{|c|c|c|c|c|c|}
\hline $\begin{array}{l}\text { Determination } \\
\text { of... }\end{array}$ & Tube insert & Reading $\left(\mathrm{mCi}^{\star}\right)$ & $\begin{array}{l}\text { Calibration } \\
\text { factor }\end{array}$ & $\begin{array}{l}\text { Corrected } \\
\text { reading }\end{array}$ & $\begin{array}{l}\text { Percent } \\
\text { deviation }\end{array}$ \\
\hline \multirow[t]{7}{*}{ Calibration factors } & Black only (source holder) & 209 & 1.0000 & & \\
\hline & Black and red & 120.30 & 1.737 & & \\
\hline & Black and orange & 66.50 & 3.142 & & \\
\hline & Black and yellow & 19.40 & 10.773 & & \\
\hline & Black and green & 5.59 & 37.388 & & \\
\hline & Black and blue & 1.89 & 110.582 & & \\
\hline & Black and purple & 0.45 & 465.517 & & \\
\hline \multirow[t]{8}{*}{ Dose calibrator linearity ${ }^{\dagger}$} & Black only & 124.5 & 1.0000 & 124.5 & \\
\hline & Black and red & 71.40 & 1.737 & 124.0 & $-0.24 \%$ \\
\hline & Black and orange & 40.10 & 3.142 & 126.0 & $1.01 \%$ \\
\hline & Black and yellow & 11.50 & 10.773 & 123.9 & $-0.49 \%$ \\
\hline & Black and green & 3.31 & 37.388 & 123.8 & $-0.60 \%$ \\
\hline & Black and blue & 1.11 & 110.582 & 122.7 & $-1.40 \%$ \\
\hline & Black and purple & 0.26 & 465.517 & 122.7 & $-1.45 \%$ \\
\hline & Mean & & & 123.9 & $-0.49 \%$ \\
\hline $\begin{array}{l}{ }^{\star} 1 \mathrm{mCi}=37 \mathrm{MBq} . \\
{ }^{\dagger} \text { Using predetermined ca }\end{array}$ & factors. & & & & \\
\hline
\end{tabular}

are absorbed in either the source container or the liner that protects the gas chamber. But $\beta$ particles create Bremsstrahlung photons, most of which have low energies (12). So measurement of a pure $\beta$-emitting source runs into the same source container considerations as a low-photon-energy $\gamma$-emitting source. If higher-energy ( $>100 \mathrm{keV}(10))$ photons are emitted, these penetrate into the gas chamber and make the source container question irrelevant. Measurement of the effect of the source container can be assessed by repeating the volume assessment above, but with a glass vial as well as a syringe and using a low-energy radionuclide, such as ${ }^{125} \mathrm{I}$.

$\alpha$-emitting radionuclides have recently become commercially available for therapeutic purposes. These pose new concerns for dose calibration. The best instruments for $\alpha$-particle detection are gas proportional counters and liquid scintillation counters, neither of which is commonly found in a nuclear medicine department. And both would require preparation of an aliquot of the radionuclide solution for measurement, a procedure with its own potential for error. The Nuclear Regulatory Commission's recommendation is that if there are not photon, $\gamma$, or $\beta$ emissions from the $\alpha$-emitting radionuclide for a dose calibrator reading, one should use unit dosages and decay correction techniques rather than direct measurement with a dose calibrator (5).

Geometry testing is performed by the dose calibrator manufacturer before shipment to the user. Correction values are listed in the dose calibrator's operations manual and should be applied as appropriate. Additional references provide examples of how to measure geometry variations in dose calibrator measurements $(12,13)$. A summary of pitfalls related to dose calibrator use is presented in Table 3 .

\section{SURVEY METERS}

Another kind of small instrument used in the nuclear medicine department on a daily basis is the survey meter. These come in a variety of designs, each of which has its own intended use (Fig. 3). Similar to the dose calibrator, the part of the detector interacting with radiation is a gas chamber. Photons ionize the gas molecules, releasing electrons that are detected via simple electronic circuits.

Geiger counters operate at high electric potential and create a voltage pulse (called a count) for each detected $\gamma$ ray, generating a readout in counts per minute (cpm). This number can be converted into a radiation field strength unit

TABLE 3

Pitfalls in Use of a Dose Calibrator

\begin{tabular}{|c|c|}
\hline Type of use & Pitfall \\
\hline \multirow[t]{7}{*}{ Routine } & Source not centered in dipper \\
\hline & Contamination of dipper or liner \\
\hline & Incorrect isotope button selected \\
\hline & Extraneous source affecting reading \\
\hline & $\begin{array}{l}\text { High voltage not as needed for correct } \\
\text { operation }\end{array}$ \\
\hline & $\begin{array}{l}\text { Negative reading due to rezeroing when } \\
\text { contamination is present }\end{array}$ \\
\hline & $\begin{array}{l}\text { Not enough time allowed for reading of } \\
\text { low-activity source }\end{array}$ \\
\hline \multirow[t]{4}{*}{ QC } & $\begin{array}{l}\text { Required QC testing not performed in } \\
\text { regulatory time frames }\end{array}$ \\
\hline & Inconsistent geometry for $\mathrm{QC}$ testing \\
\hline & Incorrect correction factors (linearity testing) \\
\hline & $\begin{array}{l}\text { Incorrect source information (constancy, } \\
\text { accuracy testing) }\end{array}$ \\
\hline
\end{tabular}


FIGURE 3. Survey meters. (A) Small Geiger counter intended for personal use. (B) Geiger counter with pancake probe, used to locate areas of radioactive contamination. (C) Geiger counter with GeigerMüller tube attachment, commonly used to monitor ambient radiation levels. (D) Ionization survey meter. This instrument operates at lower electric potential than Geiger counter, making it less sensitive and therefore not appropriate for identification of areas of contamination. ( $A$ and $B$ courtesy of Capintec, Inc.; $C$ and D courtesy of Biodex Medical Systems, Inc.)

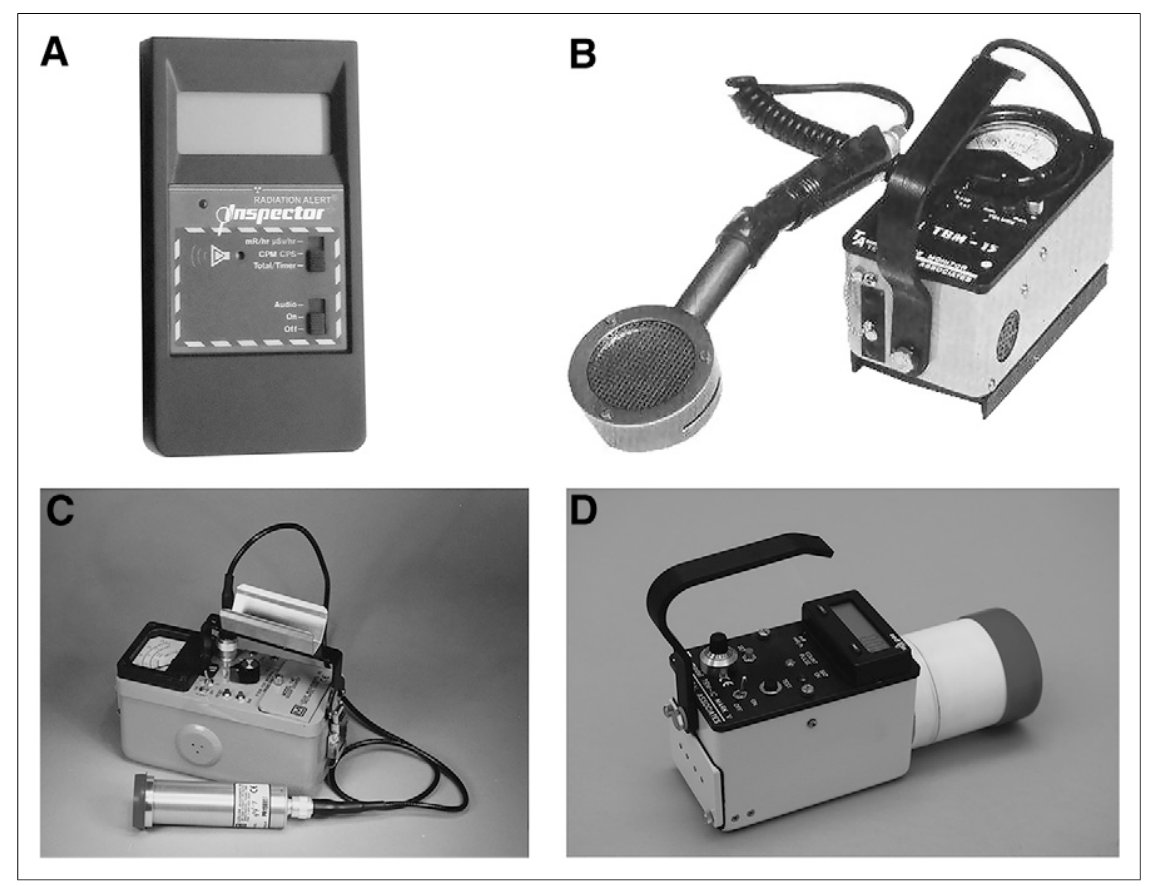

of roentgens/h, and most Geiger counters have both scales. Some types of Geiger counters are composed of a single unit, capable of being carried in one's pocket as a personal detector (Fig. 3A). The actual detector of most larger Geiger counters is usually either a Geiger-Müller tube or a pancake probe (Figs. 3C and 3B, respectively). The pancake probe is specifically designed to be used to look for radioactive contamination on surfaces such as countertops. The Geiger-Müller tube is used to measure radiation fields in a variety of situations.

An ionization survey meter (Fig. 3D) operates at a lower electric potential. It is more like a dose calibrator, in that many of the ionized electrons created by photon interactions are collected and measured as electric current. It requires a higher radiation field strength to produce a steady reading and is calibrated only in units of roentgens/h. An ionization survey meter is the right tool for measuring the radiation level of a radioactive patient, but not for contamination surveys. "Cutie pie" is a common generic name for this type of survey meter.

Given their different functions, one obvious error would be to use the wrong survey meter for a given task. But these instruments are subject to several additional pitfalls in regard to their proper use. Survey meters of either type are powered by a battery and will not generate a signal if the battery is not producing the right amount of voltage or current. This usually occurs because the meter was not turned off after a prior use, causing the battery to run out. The pancake probe or Geiger-Müller tube of a Geiger counter is usually connected to the electronic unit by a cable, and cable wires can break, causing erratic operation. Distance and extraneous-source issues also come into play with survey meters. A radiation source will not generate a signal if the detector is too far from it, but it will register any radiation source that is nearby. Contamination of a survey meter usually makes it unusable until the radioactivity has decayed.

\section{Thin End-Windows}

Both ionization survey meters and Geiger counters often have a thin end-window made of Mylar (DuPont Teijin Films) or very thin mica that allows them to register particulate $(\alpha / \beta)$ radiation; if this is damaged such that the gas chamber is no longer at the correct operating pressure, the detector will likely not function. On the other hand, most survey meters have some kind of covering to protect this fragile surface. A measurement made with the covering in place over the thin end-window will generally not include any particulate emissions. If one desires to measure $\alpha / \beta$ radiation as well as $\gamma$ radiation, the covering needs to be removed.

\section{Time Constant}

Geiger counters have a couple of additional pitfalls beyond those it shares with the ionization survey meter. One is that the meter's response to a radiation source is controlled by an electronic device called a time constant. Commercial Geiger counters may have a time constant with 2 settings (usually labeled "fast" and "slow") or a potentiometer knob allowing the time constant to vary over a continuous range. The operator must take into account the meter response time when making a measurement. A meter with a slow time constant will not respond quickly, necessitating a slow sweep of the detector. A fast time constant is preferred for many nuclear medicine tasks, such as checking gloves before putting them into a trash can. 


\section{Dead Time}

A second pitfall of the Geiger counter is dead time. The pulse of electric voltage created in response to a photon interaction comes from an avalanche of about a billion electrons that are ionized from their molecules. Once this avalanche of electrons has been released, the gas in the chamber is essentially unresponsive to new photon interactions until the electrons and ionized gas molecules recombine and return the gas to electric neutrality. This results in a time during which the Geiger counter cannot register new photon interactions. In the presence of a high-strength radiation field, a Geiger counter can experience paralysis due to dead time, in which case the measured radiation level is less than the actual exposure rate (14). One can verify that dead time is affecting a radiation measurement by moving toward the radiation source. The inverse-square law says that the intensity of the radiation field should increase exponentially as distance decreases; if the Geiger counter's readings do not bear this out, then the possibility of dead time should be considered.

\section{QC}

Daily QC for a survey meter consists of checking the battery level and verifying normal function with a radioactive source. Some meters have a built-in check source, whereas others require the use of a separate source. Whichever is the case, the reading should always be performed with the same geometry. Day-to-day fluctuations should typically be less than $\pm 10 \%$. Annually, survey meters must be calibrated to ensure that the readings accurately reflect radiation field strength. Calibration is commonly done by a health physicist rather than a nuclear medicine technologist; the procedure is completely described in the Nuclear Regulatory Commission's guidelines for medical licensees (5). Because radiation inspectors and accreditation agencies expect the calibration interval to be no longer than $1 \mathrm{y}$, it is important to keep an eye on the date for next calibration. Pitfalls in the use of survey meters are listed in Table 4.

\section{SCINTILLATION DETECTORS}

Detectors that use scintillation crystals such as sodium iodide have an inherent advantage over gas-filled detectors, in that they completely absorb most $\gamma$ rays and produce an electronic signal whose size or pulse height is proportional to the energy the $\gamma$ ray has deposited in the crystal. This allows the operator to set an energy window that encompasses the primary $\gamma$ ray of interest and to discriminate against photons with other energies. Scintillation crystals are found in nonimaging detectors such as organ probes and well counters (Fig. 4), as well as in $\gamma$ cameras and PET tomographs. The output of the former is in the form of counts per minute, each count being a $\gamma$ ray whose detected energy falls within the operatordetermined energy window.
TABLE 4

Pitfalls in Use of a Survey Meter

\begin{tabular}{ll}
\hline Type of use & \multicolumn{1}{c}{ Pitfall } \\
\hline Routine & Battery power insufficient for measurement \\
& Insufficient time allowed for reading to settle \\
& Cable wires or thin end-window broken \\
& Covering of thin end-window not removed \\
& for particulate radiation measurement \\
& Unit contaminated with radioactivity \\
& High radiation source causing dead time \\
& Unit not close enough to source (e.g., for \\
& contamination survey) \\
& Shielding material between source and \\
& detector \\
& Geometric configuration for operational \\
& check not according to protocol \\
QC & Not calibrated within regulatory time frame \\
\hline
\end{tabular}

\section{Calibration}

Scintillation detectors work by generating visible- or ultraviolet-light-range photons called scintillation photons in response to high-energy photons and then converting those into an electronic signal by means of a photomultiplier tube (PMT). The greater the energy absorbed by the scintillation crystal, the greater the number of scintillation photons generated, causing a larger output signal from the PMT. The electronic components of the scintillation detector can thus register the energy of each detected event. This is usually done in the graphic form of an energy spectrum using a multichannel analyzer (Fig. 5). Each channel on the $x$-axis of the graph represents a small energy range. In most cases, the rightmost peak seen on the energy spectrum, called the photopeak, represents the total energy of the $\gamma$ ray being measured. The $y$-axis value for each channel represents the number of events registered in that channel.

However, the PMT output can also be varied by adjusting the voltage applied to the PMT, which changes the amount of multiplication of the electronic signal within the PMT. In other words, the graph in Figure 5 can be stretched or compressed along the $x$-axis simply by changing the voltage to the PMT. Hence the determination of a proper setting for the PMT's voltage, called calibration, is essential to getting correct results. The procedure uses the $662-\mathrm{keV}$ photopeak of ${ }^{137} \mathrm{Cs}$ to establish the relationship between photon energy and the scintillation detector's output. The energy spectrum is generated, and then the high voltage is tweaked so that the photopeak aligns with some specific channel, thereby providing a direct correlation between $\gamma$-ray energy and a given channel of the multichannel analyzer. Some systems require calibration on each day that the scintillation detector is used, whereas other systems do not; manufacturer recommendations should be followed. An improperly calibrated scintillation detector will produce (at best) questionable measurements. One should always examine the energy spectrum when making a clinical mea- 


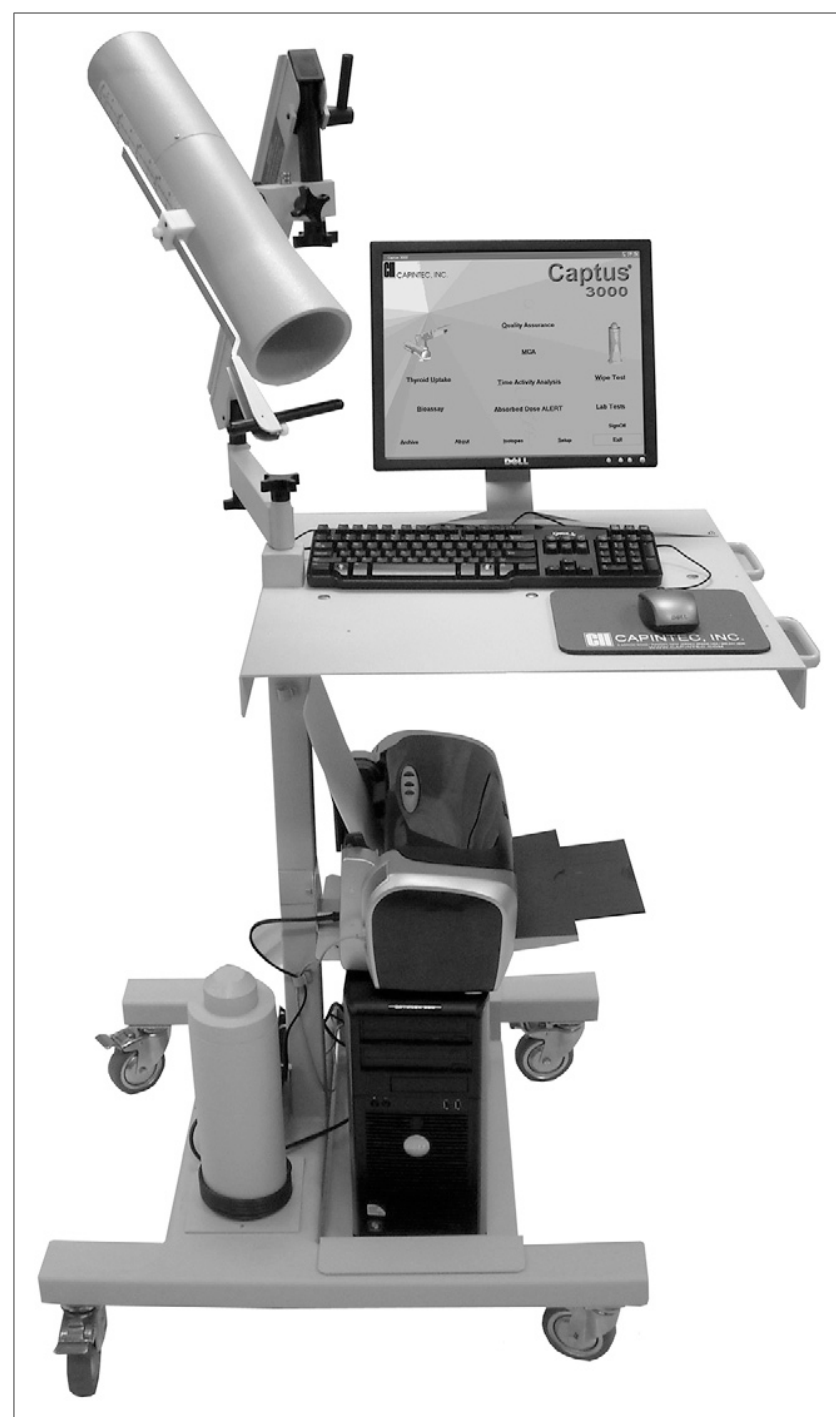

FIGURE 4. Thyroid probe/well counter. This instrument contains 2 detectors, organ probe at upper left and well counter at lower left, sitting atop base/wheel assembly. Both detectors use sodium iodide, a scintillation crystal, as the detection material, and both produce an energy spectrum as their output. Scintillation detectors have an inherent advantage over gas-filled detectors, in that the detecting material is much denser and therefore more likely to interact with $y$ rays and other high-energy photons. (Photo courtesy of Capintec, Inc.)

surement, to verify that the $\gamma$ rays of interest are registering within the preset energy window.

\section{Dead Time}

Like Geiger counters, scintillation detectors experience dead time after each detected $\gamma$-ray event. Many modern detectors are inactive during the time required to process an individual pulse, such that the system is insensitive to additional pulses during this period; thus some counts will be lost. Therefore, we need to distinguish between real time (actual clock time of a measurement) and live time (the time in which the detector is actively counting), the difference between the two being the total dead time that has been incurred for this measurement (Fig. 5). The accuracy and precision of measurements are compromised when dead time greater than about $15 \%-20 \%$ of real time is being incurred.

\section{Statistical Fluctuations}

When a radioactive source is counted several times by a scintillation detector, the cpm value is not constant but changes with every measurement, within a narrow range. This behavior is related to random fluctuations in the number of disintegrations occurring in a minute and in the number of those that are registered by the detector. Statistical behavior can be modeled, and these models can be used to evaluate the reproducibility of scintillation detector measurements. A $\chi^{2}$ test (9) is used to determine whether a detector is responding as expected from a statistical perspective. For example, a detector with normal function with regard to statistics will be out of compliance on the $\chi^{2}$ test if dead time is affecting measurements. Good practice calls for performing this test quarterly.

\section{Efficiency}

The scintillation detector measurement in units of cpm correlates to activity present in the sample or organ being counted. In many clinical measurements, only a relative measure of radioactivity is needed, but in a few situations (e.g., thyroid bioassays or weekly wipe tests) the exact relationship needs to be specified. The correlation is determined by measuring the efficiency of the detector for a source of known activity, expressed as measured cpm divided by actual disintegrations per minute (9). This efficiency factor is dependent on the measurement parameters to which it will be applied, including both the geometry and the energy window to be used in the measuring situation.

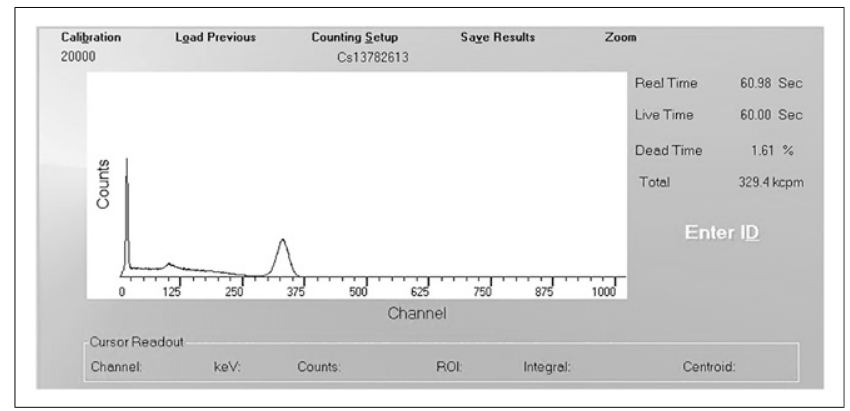

FIGURE 5. Energy spectrum. This screenshot from system in Figure 4 shows energy spectrum for ${ }^{137} \mathrm{Cs}$. $x$-axis of graph is channel number, with each channel representing small portion of energy scale. $y$-axis indicates number of counts registered in each channel. Given that energy of ${ }^{137} \mathrm{Cs}$ photopeak is $662 \mathrm{keV}$, and location of photopeak on graph is at about channel 330, each channel is approximately $2 \mathrm{keV}$ wide. Note also real-time and live-time numbers in upper right. Difference between them represents dead time incurred during this measurement, which is expressed as percentage of live time. (Photo courtesy of Capintec, Inc.) 
For example, wipe test swabs might be put into test tubes and counted in a well counter with a broad energy window. The efficiency factor must therefore be determined using a radioactive source that fits into the well counter like a test tube and with the same wide energy window. The measured cpm (after background subtraction) on each wipe test swab is divided by the efficiency factor, giving a result in disintegrations per minute. Efficiency factors should be verified at least annually. Applying an efficiency factor erroneously (e.g., a probe efficiency factor to a well counter measurement or vice versa) can result in a drastically incorrect value.

Related to this is the concept of minimum detectable activity (MDA), which is the smallest amount of radioactivity (measured in disintegrations per minute) that can reliably be detected by an instrument for a specific radionuclide or energy range. For potentially dangerous radionuclides, the Nuclear Regulatory Commission requires medical licensees to document that their detection instruments are sensitive enough to reach the lowest level of concern (5). The MDA of the scintillation detector must be at or below this regulatory limit.

The value of the MDA is dependent on the room background and the measurement time, as well as the efficiency factor for the radionuclide in question (8). Thus, the MDA value will be incorrect if the efficiency factor is determined incorrectly or if background is measured using an incorrect energy window setting. The MDA of a well counter or probe should be verified annually, in conjunction with efficiency factor determination, for each radionuclide of regulatory concern. In most nuclear medicine departments, the radionuclide of greatest concern is ${ }^{131} \mathrm{I}$, but if a lab is doing ${ }^{90} \mathrm{Y}$ therapies it should have an MDA value for that isotope as well.

Let us revisit for a moment the distinction between Geiger counters and scintillation detectors for detection of contamination. Sensitivity is defined as the ability to detect low levels of activity. Geiger counters are able to register individual $\gamma$ rays, hence indicating contamination, but they are not able to quantify the amount except with a very slow time constant and a long-duration measurement. Scintillation detectors are considerably more sensitive than Geiger counters. At the regulatory lower limit of $37 \mathrm{~Bq}(1 \mathrm{nCi})$, a scintillation detector can give a statistically precise answer in 1-3 min, whereas a Geiger counter might require 5-10 min to give the same kind of precision.

\section{QC}

Routine QC measures for scintillation detectors include daily, quarterly, and annual testing (6,7). Daily, one should calibrate the detector (often done automatically on newer detectors) and perform a constancy check (verifying that the reading of a reference source is similar to prior days' values). Quarterly tests include energy resolution using the full width at half maximum (measure of the width of the ${ }^{137} \mathrm{Cs}$ photopeak (2)) and $\chi^{2}$ test. Determination of the efficiency factor should be performed annually. Many QC tests are automated on modern scintillation detectors.

The most common errors in the use of scintillation detectors relate to the basic issues of radiation detection discussed earlier: geometry, presence of extraneous radiation sources, and energy discrimination. Any inconsistencies in geometry such as source-detector distance or even positioning of the source will produce inconsistent measurements. Choosing the wrong reference source or entering reference source information incorrectly will likewise create erroneous QC results. Application of an incorrect energy window will always give erroneous results. Background must be measured and subtracted, especially when detection of low levels of radioactivity is the goal. Contamination of the detector or the presence of extraneous radiation sources will certainly lead to incorrect numbers. Well counter measurements in particular are subject to dead-time issues, such that the upper limit of radioactivity that should be counted in a well counter is about $30-40 \mathrm{kBq}(1 \mu \mathrm{Ci})(8)$. Organ probes, because of differences in counting geometry, can measure much higher activity levels before demonstrating dead-time effects. Sources of error in the use of scintillation detectors are summarized in Table 5.

\section{SURGICAL PROBES}

The last category of small instruments to be discussed is the intraoperative probe used for sentinel lymph node

TABLE 5

Pitfalls in Use of a Scintillation Detector

\begin{tabular}{ll}
\hline Type of use & Pitfall \\
\hline Routine & Geometry of measurement not according \\
to protocol & Calibration performed incorrectly \\
Incorrect energy window chosen & Extraneous radiation source present \\
(patient, contamination, etc.) \\
Background not subtracted \\
Source activity too high; measurement \\
incurs dead time \\
Efficiency factor not applied when needed \\
Incorrect detector chosen (well counter \\
vs. thyroid probe) \\
Daily calibration not performed \\
or performed incorrectly \\
Geometry of constancy measurement \\
not according to protocol \\
$\chi^{2}$ test affected by extraneous sources \\
of radiation \\
Efficiency factor out of date or not \\
determined according to protocol \\
Current source activity not calculated \\
correctly for efficiency factor \\
determination \\
Energy window incorrect for given \\
QC test \\
\\
\hline
\end{tabular}




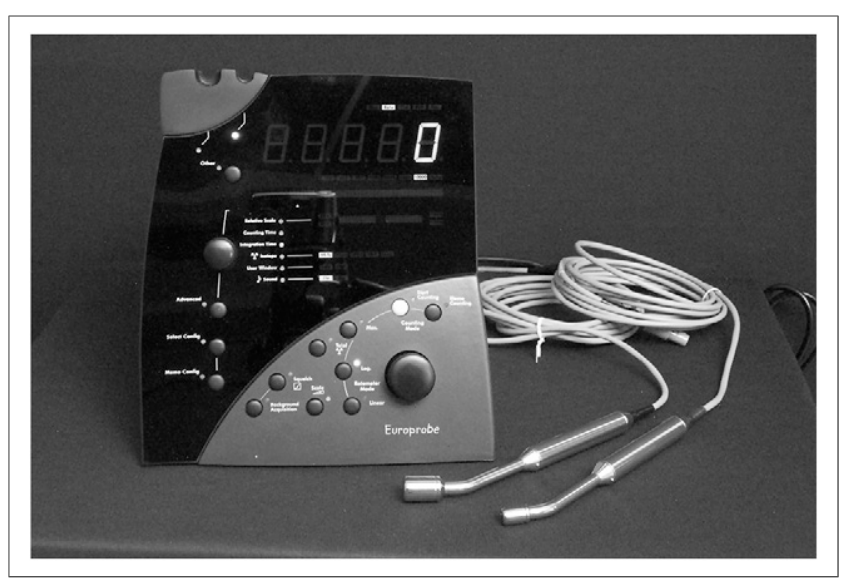

FIGURE 6. Surgical probe. This instrument is designed to identify small foci of radioactivity such as lymph nodes in operative setting. The detecting material in this case is semiconducting material, cadmium-zinc-telluride. Semiconductor radiation detectors interact with photon or particulate radiation in ionization interactions, creating electrons that are sent directly into electronic circuit. No bulky PMT is required as in organ probe shown in Figure 4, making this a better instrument for surgical operation. (Photo courtesy of Capintec, Inc.)

identification for patients with breast cancer, melanoma, and other types of malignancies (Fig. 6). In some settings, the surgical probes are kept in the nuclear medicine department and are delivered to the surgery suite as needed; in others, they are owned and maintained by the surgery department.

Intraoperative probes function much like organ probes made with sodium iodide, but the active volume is a semiconducting material such as cadmium-zinc-telluride rather than a scintillation crystal. Semiconductor radiation detectors do not require a PMT; they can be connected directly to an electric circuit, which greatly reduces their bulk. The active area of the detector is in most instruments surrounded by a collimator, making the probe highly directional. Activity that is not within the probe's line of sight may go undetected. Detection of radioactivity within the preset energy window causes both a visual and an audible response. The level of response can be modified using sensitivity settings on the instrument; these should be set according to the surgeon's preference. Semiconductor

TABLE 6

Pitfalls in Use of a Surgical Probe

\begin{tabular}{|c|c|}
\hline Type of use & Pitfall \\
\hline \multirow[t]{4}{*}{ Routine } & Incorrect energy window chosen \\
\hline & Audible signal level not adjusted correctly \\
\hline & Directionality of probe not considered \\
\hline & Probe not properly cleaned and sterilized \\
\hline \multirow[t]{2}{*}{ QC } & $\begin{array}{l}\text { Daily constancy test not performed with } \\
\text { correct energy window }\end{array}$ \\
\hline & Battery power insufficient for probe operation \\
\hline
\end{tabular}

detectors and scintillation detectors are about equally sensitive to $\gamma$ photons.

Because semiconductor radiation detectors, like scintillation detectors, can distinguish photon energies, an energy window must be specified. Semiconductor materials have a much finer energy resolution than scintillation detectors, allowing use of a narrower window. Hence one potential operational error is that if an incorrect energy window is set, a lesion containing radioactivity may not register in the window. Before use in surgery, the probe should be calibrated and a constancy test performed, using the energy window setting for the nuclide to be detected in the surgical procedure (15). An incorrect energy window setting is the most common problem with these detectors.

After its use in a surgical procedure, the probe is disconnected from the electric unit and then cleaned and sterilized according to the manufacturer's specifications (15). Because these units are battery-powered, recharging the battery after use is essential. The instrument should be stored at room temperature and should not be subjected to large temperature changes. The most significant pitfall would be to deliver the unit to the surgical suite in a condition in which it is not performing properly. Table 6 summarizes the potential ways a surgical probe can be misused.

\section{CONCLUSION}

The small radiation-detecting instruments discussed in this article are less complex than the imaging equipment used in nuclear medicine. But they can be misused in several ways. The manufacturer's recommendations for use should be incorporated into the department's QC protocols, as should regulatory requirements and community standards of practice $(6,7)$. Routine QC is extremely important, as it allows verification of the detector's integrity and identifies potential malfunctions. In an ideal world, attention to routine QC will allow problems to be found and addressed before they affect clinical results, and vigilance in routine measurements will lead to appropriate clinical findings.

\section{DISCLOSURE}

This article originated as an educational lecture developed by Art Hall, BS, CNMT, and subsequently presented by him and by Kathy Thomas, CNMT, PET, during chapter and national meetings of the Society of Nuclear Medicine and Molecular Imaging. Art Hall and Kathy Thomas are applications specialists and technical support specialists for Capintec, Inc., and are therefore considered to have a conflict of interest in regard to authoring an article for this journal. I am pleased to present their discussion of this important topic with some additional thoughts of my own. There is no other potential conflict of interest relevant to this article. 


\section{REFERENCES}

1. Christian PE, Waterstram-Rich KM. Nuclear Medicine and PET/CT Technology and Techniques 7th ed. St. Louis, MO: Elsevier Mosby; 2012: 45-81, 189-195.

2. Prekeges JL. Nuclear Medicine Instrumentation. 2nd ed. Burlington, MA: J\&B Learning; 2013:3-47, 124.

3. Kowalsky RJ, Falen SW. Radiopharmaceuticals in Nuclear Pharmacy and Nuclear Medicine. 3rd ed. Washington, DC: American Pharmacists Association; 2011: 59-73, 78-85, 299-305.

4. Knoll GF. Radiation Detection and Measurement. 4th ed. New York, NY: Wiley; 2010:136-144, 201-216, 231-237, 345-376.

5. Howe DB, Beardsley M, Bakhsh SR. Consolidated guidance about materials licenses: program - specific guidance about medical use licenses (NUREG-1556, volume 9, revision 2). United States Nuclear Regulatory Commission website. http://www.nrc.gov/reading-rm/doc-collections/nuregs/staff/sr1556/v9/r2/. Published January 2008. Accessed February 27, 2014.

6. ACR-SNM technical standard for diagnostic procedures using radiopharmaceuticals. http://snmmi.files.cms-plus.com/docs/ACR-SNM_Technical_standards_for_ diagnostic_procedures_using_radiopharmaceuticals_1382731756580_2.pdf. Published 1994. Updated 1995, 1998, 2001, 2006, 2009, 2011. Accessed February 27, 2014.
7. The IAC standards and guidelines for nuclear/PET accreditation. Intersocietal Accreditation Commission website. http://www.intersocietal.org/nuclear/standards/IAC NuclearPETStandards2012.pdf. Updated August 2012. Accessed February 27, 2014.

8. Zanzonico P. Routine quality control of clinical nuclear medicine instrumentation: a brief review. J Nucl Med. 2008;49:1114-1131.

9. Wells P. Practical Mathematics in Nuclear Medicine Technology. 2nd ed. Reston, VA: Society of Nuclear Medicine and Molecular Imaging. 2011:112-117, 122-123, 127-128.

10. Graham LS. Nuclear Medicine Self-Study Program II: Instrumentation. Reston, VA: Society of Nuclear Medicine and Molecular Imaging. 1996:3-4.

11. Suzuki A, Suzuki MN, Weis AM. Analysis of a radioisotope calibrator. J Nucl Med Technol. 1976;4:193-198.

12. Valley J-F, Bulling S, Leresche M, Wastiel C. Determination of the efficiency of commercially available dose calibrators for $\beta$-emitters. J Nucl Med Technol. 2003;31:27-32.

13. Santos JAM, Dias AG, Bastos AL. Characterization of geometric uncertainties of a dose calibrator during measurement of ${ }^{90} \mathrm{Y}$ activity. J Nucl Med Technol. 2011;39:125-130.

14. Rollo DF. Nuclear Medicine Physics, Instrumentation, and Agents. St. Louis, MO: CV Mosby; 1977:205-206.

15. Halkar RK, Aarsvold JN. Intraoperative probes. J Nucl Med Technol. 1999;27: 188-193. 